\title{
Daylighting Strategies: Building's Sustainability and Energy Efficiency
}

\author{
Barbara Gherri \\ Department of Civil, Environmental and Land Engineering and Architecture, University of Parma, Parma 43124, Italy
}

\begin{abstract}
Improving daylighting strategy is a mandatory step to achieve visual enjoyment and energy saving in buildings. Psycho, physiological effects and energy performance have to be investigated in order to define a range of different daylighting strategies, thanks to daylighting devices and climate based daylight modeling. Daylighting optimization ensures indoor healthier rooms, reduces electric light consumption and cuts the risk of glare. The best way to achieve these targets is to define users lighting needs, based on visual targets and to draw up some green measures to reduce electricity demands. Involving new climate-based daylight modeling metrics aims at defining proper illumination targets, in order to drastically reduce electrical lights, as well as reducing thermal loads deriving from cooling and HVAC (heating, ventilation and air conditioning) systems.
\end{abstract}

Key words: Energy saving, daylight rating system, sustainability in buildings, climate based daylight modeling.

\section{Introduction}

According to the latest policies, to promote low carbon solutions and to avoid the depletion of natural resources, in case of new buildings and in refurbishment action, one of the most important strategy is to consider daylight as a mean to maximize the entry of sunlight, since an excess of solar radiation can cause, depending on latitude and orientation, overheating problems, to exploit the benefits in terms of energy and indoor health.

The largest energy consumption in buildings, especially in medium latitude regions, like Mediterranean and southern European area, is due to heating, cooling and electric appliances, consequently daylighting design, as well as insulation and thermal refurbishment, can dramatically reduce heat losses. Italian homes annually consume about $160 \mathrm{kWh} / \mathrm{m}^{2}$ and about $14 \%$ is related to light demands (Table 1).

It is also well known that maximizing the sunlight

Corresponding author: Barbara Gherri, M.Arch, Ph.D., research fields: daylight architecture, daylight and thermal assessment, sustainable and technological design in residential buildings, low carbon strategies in urban microclimate for outdoor thermal comfort evaluations. E-mail: barbara.gherri@unipr.it. penetration in indoor spaces can be very effective in terms of energy savings, to cut off electricity consumption and in terms of thermal loads reduction, especially in Mediterranean regions.

The potential amount of energy saved by effective daylighting design should involve a wide range of practitioners, to an integrated approach to daylight planning and management.

Although the design and constructive premises are clearly oriented toward the definition of multidisciplinary strategies to help saving energy, the main issue to deal with is succeeding in an efficient and green integration of daylighting strategies in each design step, involving retro commissioning actions.

A widespread belief considers sunlight as a satisfactory instrument itself to address and to solve several energy problems, but proper daylight design should involve a wide range of considerations: building orientation, inner surface materials, visual tasks and shading devices. Energy saving potential in electric demand has been usually evaluated as the amount of daylight factor levels to illuminate indoor space, according to Hunt [1] in 1970. Because of everyday experience and applied calculation have largely revealed that daylight can replace up to $50 \%$ of 
Table 1 Average Italian homes consumption.

\begin{tabular}{lc}
\hline Energy & Average homes consumption \\
\hline Heating & $64 \%$ \\
Cooling & $16 \%$ \\
Domestic hot water & $6 \%$ \\
Lighting & $14 \%$ \\
\hline
\end{tabular}

the energy used in buildings, the very first attempt to address the point is make manual switching for electrical appliances more effective. Users behavioral patterns, either for large working spaces or for public buildings, have also been used for years as a simply tool to assess energy saving potential. Since lighting, heating and cooling techniques are deeply inter-reliant and, unless wisely planned, too much daylight can bring with it undesirable heat gains, it is now clear that a too simplistic approach, as well as too many limitations, can impair an effective daylight assessment.

Any conscious energy saving approach needs to consider daylighting and shading systems, as well as electric lighting at the same time. Some basic attempts to provide automated control for dimming artificial light, according to user requirements, were proposed in public buildings, thanks to a wide range of theoretical formula and criteria, that mostly considered users' behavior patterns and sun path as the main drivers.

Design of complex systems for automated lighting control has been significantly improved over the last thirty years thanks to the so-called POE (Post Occupancy Evaluation) tests, which can detect a considerable amount of shortcomings, to be applied during the design phase or later on. Nevertheless, this approach is still too simplistic to address a complex issue.

\section{Daylighting Appraisal to Ensure Energy Saving Performance}

In order to constraint energy requirements, the use of daylight with the integration of artificial light is an essential step to achieve. Besides direct savings, it is widely demonstrated that a proper daylight evaluation can reduce energy consumption for lighting, as well as indirect energy savings can be obtained in medium latitude, thanks to reduced heat production and energy consumption by air conditioning. An effective daylight assessment should involve energy saving strategies, deriving from the design and the running phase, thanks to some appropriate techniques and design solutions, to be applied in existing building and new ones. One of the most effective daylight assessments should therefore involve CBDM (climate-based daylight modelling) that can evaluate the indoor energy savings potential, by maximizing daylight penetration in winter and by shading it in summer.

So far, the most common procedure to allow a well-daylit environment, according to users' needs and to local climate, aims at providing some basic solar shading during daylit hours, as well as at providing a proper protection from glare, especially for windows areas and redirecting sunlight in the depth of the room, in order to ensure the most homogenous environment. The methodology proposed lately aims at developing the new dynamic parameters, according to CBDM model, in order to encompass outdoor climatic conditions that change continuously.

\subsection{Daylighting Factor Shortcomings: The Demand for a New International Paradigm}

The standard procedure to assess the actual daylight dose in a room has involved simplified models, tables, diagrams, nomograms, and some complex scale models, to be used under artificial or real sky. Among all these tools, the most reliable and easier method to assess daylight percentage so far is the DF (Daylight Factor) accordingly to the static approach.

The daylight factor is therefore the most accustomed method to measure illumination levels, according to static parameter, DF states the ratio between illuminance on the work plane and the illuminance available outdoor, in case of overcast sky. 
Static daylight assessment involves therefore a too simplistic approach, because of snapshot or single-point-in-time method that does not consider direct radiation from the sun. DF does not measure good quality of daylight, but is a parameter for evaluating the satisfaction of a minimum quantity of natural light. Though it is easy to calculate, it considers the worst sky conditions with overcast luminance distribution and disregards the changing sky conditions all year round. The main limitation of DF method regards the fact that it is a geometrical parameter, it can be calculated for a day at one time and it does not take into account the geographical location, as well as solar exposure. Average daylight factor is apparently inadequate to evaluate qualitative aspects, such as natural light distribution, luminance ratio and view, as well as other related limitations in predicting glare and energy saving measures.

\subsection{CBDM Approach and Climate Based Related} Metrics

Therefore, the CBDM [2], although not yet widely accepted, is now to outline the simulation of natural light distribution in a room. This model aims at providing an accurate method to evaluate whether a daylit building meets minimum standards for energy and users comfort performance. Static DF metric does not account for the temporal and spatial aspects of daylight, nor of occupants' comfort, involving a large amount of electricity to respond users' expectations. New daylight dynamic method-DDS (Dynamic Daylight Simulation)—has been developed to overcome the limitations of daylight factor and considers some meaningful issues (windows' position, orientation of building envelope and the fluctuating amounts of light over the day and over the year). Contrary to DDS, CBDM considers that luminance and glare change over the year, according to the outdoor daylight availability and to prevailing climate conditions, making it necessary to assess a large amount of data.
The dynamic metrics developed so far, gives evidence to the aptitude of a room to reach a minimum level of illuminance, thanks to daylight exclusively. The most innovative metrics (Fig. 1) according to CBDM are DA (daylight autonomy) and UDI (useful daylight illuminance) [3].

DA shows the percentage of yearly occupied time, during which a certain light level is reached through the use of daylight only, considering all sky conditions that occur yearly during daytime through weather data files.

DA calculation is also useful to quantify the saturation of daylight in an environment and to determine the percentage of direct sunlight.

UDI can be considered as a goal-oriented metric, which tells about how target illuminances are spatially reached.

UDI assesses the annual occurrence of illuminances, considering a certain area, which is within the useful fixed range of illuminances. UDI distinguishes the illuminances lower than $100 \mathrm{~lx}$, that are not enough to accomplish visual tasks, so extra artificial light is required, between $100 \mathrm{~lx}$ and 2,000 lx, that are

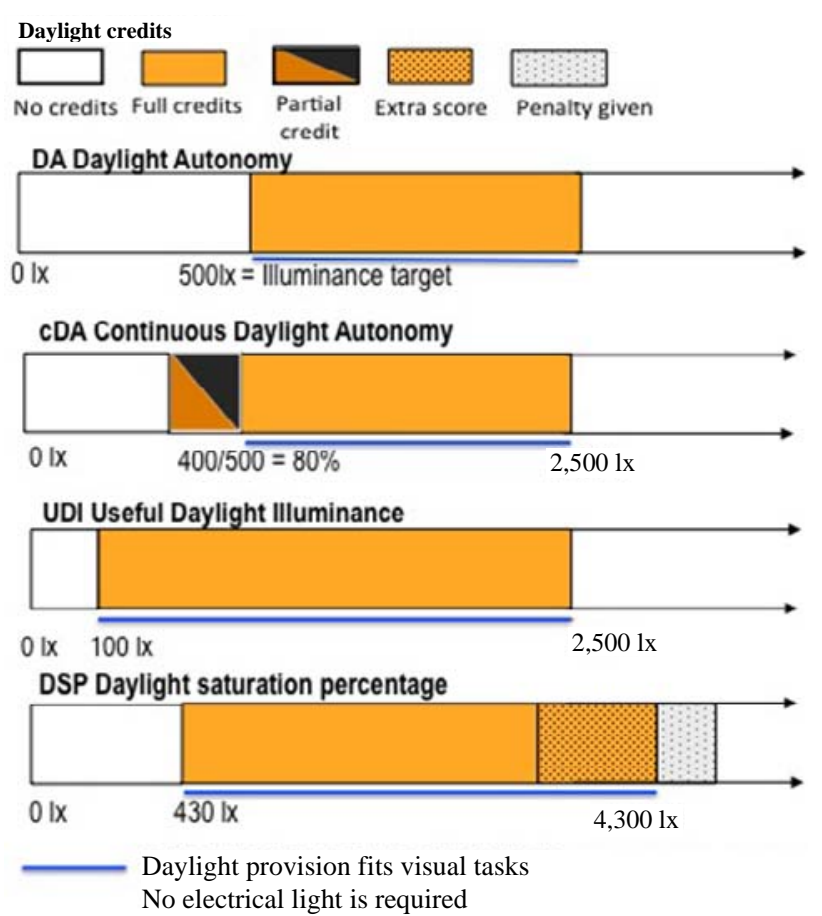

Fig. 1 Daylight metrics. 
considered as useful and superior to 2,000 lx, which are too high and can lead to glare and overheating.

DSP (Daylight Saturation Percentage) is an adjustment of UDI that increases the lower limit to $430 \mathrm{~lx}$ as well as the upper limit is set to 4,300 lx.

Provision of adequate levels daylight illuminance is known to affect the total amount of artificial light required. Unlike the steady state approach, DDS method provides integration between natural and artificial lighting components and, in case some illuminance target is not achieved, the new criterion expects the introduction of variable amounts of artificial luminaries, providing a practical instrument to predict when and how much artificial light is needed.

UDI and DA retain in their formulation the effectiveness of the static approach simplicity-typical of daylight factor approach, thanks to new illuminance targets, which are representative of illumination levels achieved and that can provide crucial information, the concurrency of discomfort conditions and visual overheating near windows. Unlike DA, UDI gives for the first time a full role to those illuminances that are lower than the usual threshold of $500 \mathrm{~lx}$, making a significant benefit in terms of energy savings, showing that lower illumination levels can still contribute in some visual tasks, with no need to enhance artificial light levels. It is clearly demonstrate that UDI analyses can help to cut down artificial light provision, helping to save energy.

Therefore, the inversely proportional relationship between UDI and power consumption is a key indicator of the potential of CBDM in energy savings actions.

In a very innovative way, new CBDM [4] approach is tailored to respond to occupants needs and to enhance energy saving potential linked to daylight provision.

The new metric acknowledges that even a partial contribution of daylight to illuminate a space is still beneficial. In case it is not possible to reach a threshold by the only provision of daylight, it is easy to foresee how much artificial light is therefore required. CBDM metrics are also outlined as human-factors based metrics, because of the combined approach they offered, that is related to variable provision of artificial lighting and the fluctuating users. To manage several variables, parameters and large amount of climatic and illuminance data, some softwares are currently available to carry out daylight simulations and to handle an energetic assessment.

Software as VELUX Energy and Indoor Climate Visualizer, Energyplus [5] and Ecotect, let the user to arrange each geometrical and optical building features, considering the main visual task and providing detailed reports and comparison to calculate different technological solutions (toplight, corelight and sidelight), considering shading devices, rooftop sheds or inner light courts, since visual transmission data, shielding elements, blackout and opaque solutions. Most of these softwares can embody natural light and natural ventilation rates, and they can estimate artificial light doses, as they foresee how many tons of $\mathrm{CO}_{2}$ can be reduced or increased. Thanks to these dynamic tools, natural ventilation and natural light become simple, intuitive by virtue of animations.

\subsection{Daylighting Strategies in Energy Assessment Procedures}

The increasing attention towards the daylighting issues and their related benefits leads to the definition of some specific targets to reach in case of standing and new buildings, to get some satisfactory energy standards [6]. The main goal of these standards and rating systems is to establish conventions and procedures for the evaluation of energy requirements of lighting in buildings, to provide a global procedure to define a numeric indicator of energy performance.

Because of the benefits of daylit interiors are well known, almost every kind of energy and 
environmental quality assessment tools include daylight performance as a measure of health and well-being. A large number of accreditation procedure and building standards involve both volunteer rating methods and compulsory ones, whose strict application can guarantee and influence design process and building management. Among these, the first to include a daylighting assessment in its appraisal is the U.S. LEED (Leadership in Energy and Environmental Design) system.

Designed in 1999 by the U.S. Green Building Council, LEED is now used to certify almost every kind of building types, according to their functions. The LEED Rating System is therefore designed to identify the most effective strategies to achieve low carbon buildings and to get to the best solution in case of retrofit and new construction, for a large variety of building types. Thanks to daylighting usage each building can earn a maximum of two credits. According to LEED [7], a credit is given in case a minimum daylight factor of $2 \%$ is achieved with no regards to direct sunlight component in $75 \%$ of room, according to a specific visual task.

LEED 8.1 also gives a credit in case of external view is assured by the presence of large windows with transparent panes [8].

Even if LEED gives a score only in case of $75 \%$ of internal space is affected by daylight, it is possible to assign partial credits. In case of software evaluation, it is compulsory to get a daylight illumination level of 25 foot-candles in minute of $75 \%$ of frequently working areas, to earn an extra credit there must be, at least, one window with transparent pane, to get an outside view (90\% of window area). LEED system aims at promoting an effective daylighting design or retrofit action, thanks to suitable buildings orientation, to make the best use from solar energy for heating or daylight, as well as to encourage natural ventilation and passive cooling, strategies that help reducing heat gain and control glare and contrast. A similar approach can be found in British BREEAM (Building
Research Establishment Environmental Assessment Method) that uses English building codes to give different range of scores to earn points. There is a considerable overlap between the two rating systems. The targets set in BREEAM are mainly linked to the presence of some technological elements, whilst LEED is less prescriptive, letting designers choosing among architectural and technological issues.

The former is more focused on inhabitant comfort and internal pollution issues, whilst BREEAM requirement for daylighting achievement is quite onerous. According to the latter, at least $80 \%$ of floor area in occupied spaces must have an average daylight factor of $2 \%$ or more, as well as a uniformity ratio of at least 0.4 or a minimum point of daylight factor, at least $0.8 \%$.

The European Committee for Standardization [9] has recently introduced some recommendations to evaluate buildings energy performance, including artificial and natural light.

One of the newest methods to assess daylight energy saving potential is LENI (Lighting Energy Numeric Indicator) noteworthy to evaluate energetic and luminous performances in buildings. LENI metric aims at supporting the global effort to save lighting energy by means of daylighting promotion, as the very unique method to ensure good visual and thermal comfort. LENI ratio is therefore a very effective meter to underline the forthcoming energy performance of a building in standard conditions. It shows energy efficiency of the building itself and of its heating, cooling, ventilation, as well as lighting systems.

Besides, ELI (Ergonomic Lighting Indicator) is a supplementary criterion that has been added lately to provide a comprehensive assessment of indoor light quality, it is based on five parameters to appraise visual comfort conditions in a daylit environment as well as it is a useful tool to detect most likely effective energy saving strategies. 


\subsection{Daylight in Buildings, Standards and Regulations in Italy}

The current state of prescription and rating system for Italian buildings emphasizes, moreover, the regional independence in local credit systems. Although the main Italian daylighting assessment methods and energy efficiency rating system generally concern the appraisal of buildings energy requirements, they also consider the primary energy demand for cooling and lighting.

One of the most shared standard Italian rating systems is called ITACA [10], based on GBTool, developed by Federal Association of Italian regions. It applies just both for residential and public buildings and it aims at merging the most widely shared regional certification processes into an inclusive national method. Among several indicators, there are some references to natural light, to users' visual comfort, to penetration of direct sunlight and to light consistency.

\section{Conclusions}

Architectural devices and other technology issues, with regard to daylight opportunities, offer some remarkable opportunities in reducing energy consumption for electric lighting and for thermal conditioning even if, an effective practice is still constrained by high costs, defective performance of current technologies and some diffuse misunderstandings. The main target to upcoming development and in rating systems improvement, especially for medium latitude regions should focus on the great energy saving potential using daylight as primary source of illumination, providing proper devices and convenient solutions to consciously address the sunlight, to maximize solar penetration and to support light spreading all over the room.

A remarkable example of integrated design that has fully explored the helpful contribution in terms of physical health and in terms of energy saving can be found in some recent housing prototypes, as the best known Home for Life, a project carried out by AART Architects A/S in Aarhus, Denmark. Specific attention has been paid to windows design, to create a sort of extension of indoor area toward the outdoor courtyard, so the windows area covers $40 \%$ of the floor, two times larger than traditional residences in northern Europe. Each window employs high-tech panes, designed to absorb heating excess and to convey the most of the solar radiation, to ensure a minimum level of DF never less than 2.5\%. The pitch of the roof, facing south, is made of a suitable moving retractile overhang to provide solar shading during summer time, and to let solar radiation to spread evenly in depth during winter. Shading devices, for windows and skylights, have been designed to simplify daily management, as well as, ground floor and first floor plan disposition and windows arrangements can help reduce ventilation losses up to $34 \%$.

The application of an accurate daylighting design can create a lot of benefits, from utility savings to environmental responsibility.

While new architectural trends aim, wherever possible, at answering users' needs and requirements through the integration of automated control and management of building devices, a very effective solution can be found in the dynamic management of natural light that actually considers natural paradigms of light path, during daytime and according to seasonal changes.

\section{References}

[1] D.R.G. Hunt, Improved daylight data for predicting energy savings from photoelectric controls, Lighting Research and Technology 11 (1979) 19-23.

[2] J. Mardaljevic, Examples of climate-based daylight modeling, in: CIBSE National Conference 2006 Engineering the Future, London, 2006.

[3] J. Mardaljevic, L. Heschong, E. Lee, Daylight metrics and energy saving, Lighting Research and Technology 41 (3) (2009) 261-283.

[4] C.F. Reinhart, J. Mardaljevic, Z. Rogers, Dynamic daylight performance metrics for sustainable building design, Leukos 3 (1) (2006) 1-25.

[5] W.L. Carroll, R.J. Hitchcock, Delight2 daylighting 
analysis in energy plus: Integration and preliminary user results, in: International IBPSA Conference-BS 2005, Montreal, Canada, 2005, pp. 139-142.

[6] K. Shaw, Daylight: An energy saving resource, in: Lights of India Conference, Goa, India, 2010.

[7] K. Carrier, M.S. Ubbelohde, The role of daylighting in LEED certification: A comparative evaluation of documentation methods, in: Proceedings of the 2005 Solar World Conference, Orlando, 2005.
[8] US Green Building Council, LEED ${ }^{\mathrm{TM}}$ Reference Guide version 2.0., Paladino Consulting LLC, 2006, www.usgbc.org (accessed June 1, 2013).

[9] Energy Performance of Buildings-Energy Requirements for Lighting, BS EN 15193, 2007.

[10] A. Moro, A. Bellone, G. Piccoli, Rating systems in the public and financial sectors, in: CESB 07 PRAGUE International Conference, Central Europe towards Sustainable Building, Prague, Czech Republic, 2007. 